PROCEEDINGS OF THE

AMERICAN MATHEMATICAL SOCIETY

Volume 138, Number 3, March 2010, Pages 847-852

S 0002-9939(09)10125-9

Article electronically published on October 22, 2009

\title{
ON BIMODULES OVER NOETHERIAN PI RINGS
}

\author{
AMIRAM BRAUN \\ (Communicated by Martin Lorenz)
}

\begin{abstract}
Let $R$ be a prime Noetherian PI ring, and let $I$ be an ideal in $R$ satisfying $x I \subseteq I x$ for some $x$ in $R$. We prove that $x I=I x$. This is obtained as a corollary of a similar more general result, where $I$ can be taken as any finitely generated torsion-free central $R$-bimodule.
\end{abstract}

\section{INTRODUCTION}

It was proved in 1, Proposition 1.8] that $x R \subseteq R x$ implies $x R=R x$ for each prime Noetherian PI ring $R$. This result has a long history. It was proved by Frölich et al. in [6] and by Vasconcelos in [12] in special cases. It was then conjectured by Montgomery in [10] to hold in general. This was verified by Guralnick et al. in 8] for affine Noetherian prime PI rings. It was then confirmed in [1] and was also extended to a version valid for an invertible ideal.

The following extension to bimodules is our main result.

Theorem A. Let $R$ be a prime Noetherian PI ring, and let $M$ be a finitely generated torsion-free (from both sides) R-bimodule which is $Z(R)$-central. Let $x$ in $R$ satisfy $x M \subseteq M x$. Then $x M=M x$.

The next immediate corollary appears to be new.

Corollary B. Let $R$ be a prime Noetherian PI ring, and let $I$ be an ideal in $R$ satisfying $x I \subseteq I x$. Then $x I=I x$.

This somewhat unexpected "rigidity" result triggers the question of whether $x R=R x$ also holds in this case. We show at the end of this paper that this is indeed the case if $R$ is, in addition, a maximal order.

Theorem A was partially motivated by our investigation of the Noetherian property of the $I$-adic ideal completion of a Noetherian PI ring, the results of which will appear elsewhere.

\section{Proofs}

Lemma 2.1. Let $R$ be a prime PI ring, and let $M$ be a finitely generated torsion-free left $R$-module. Then $M$ is embedded in a finitely generated torsion-free $Z$-module, where $Z=Z(R)$, the center of $R$.

Received by the editors April 18, 2009, and, in revised form, June 23, 2009, and July 19, 2009. 2000 Mathematics Subject Classification. Primary 16P40, 16R20; Secondary 16N20, 16D20.

(C)2009 American Mathematical Society Reverts to public domain 28 years from publication 
Proof. By the torsion-freeness of $M$ we identify $M$ with $R \otimes_{R} M \subset Q(R) \otimes_{R} M$. By Formanek (e.g. [11]), we have $R \subset Z w_{1}+\ldots+Z w_{s}$, where $w_{i} \in Q(R)$. Also $M=\sum_{i=1}^{r} R m_{i}$. Hence

$$
M=\sum_{i=1}^{r} R \otimes m_{i} \subset \sum_{i}^{r} \sum_{j}^{s} Z w_{j} \otimes m_{i}=\sum_{i, j} Z\left(w_{j} \otimes m_{i}\right) .
$$

The latter is a finitely generated $Z$-submodule of $Q(R) \otimes_{R} M$ and is $Z$-torsion-free since $Q(R) \otimes_{R} M$ is a torsion-free $Q(R)$-module.

Corollary 2.2. Let $R$ be a prime PI ring, and let $M$ be a finitely generated torsionfree left $R$-module. Then $\operatorname{End}_{Z}(M)$ is a prime PI ring.

Proof. $M \subseteq \sum_{i} Z v_{i j}$, where $v_{i j}=w_{j} \otimes m_{i} \in Q(R) \otimes_{R} M$ as in Lemma2.1, Let $K \equiv$ $Q(Z)$. Then if $\alpha \in \operatorname{End}_{Z}(M)$ we define, for each $\delta \in Z, \tilde{\alpha}\left(\delta^{-1} m\right) \equiv \delta^{-1} \alpha(m)$. This is clearly well defined and $\tilde{\alpha} \in \operatorname{End}_{K}\left(K \otimes_{Z} M\right)$. So the map $\alpha \rightarrow \tilde{\alpha}$ is an injection, showing that $\operatorname{End}_{Z}(M)$ is a PI ring. Let $\psi \in \operatorname{End}_{K}\left(K \otimes_{Z} M\right)$. Find $c \in Z(R)$ such that $c v_{i j} \in R \otimes_{R} M=M$, so $v_{i j} \in c^{-1} M \subset K \otimes_{Z} M$. Hence $\psi\left(v_{i j}\right) \in K \otimes_{Z} M$. So find $c_{1} \in Z$ such that $c_{1} \psi\left(v_{i j}\right) \in Z \otimes_{Z} M=M$. Hence $c_{1} \psi \in \operatorname{End}_{Z}(M)$, and consequently $\operatorname{End}_{Z}(M)$ is an order in the matrix ring $\operatorname{End}_{K}\left(K \otimes_{Z} M\right)$, implying that $\operatorname{End}_{Z}(M)$ is a prime ring.

Let $S$ be a central simple finite dimensional $K$-algebra. Then for each $x \in S$ one defines the reduced norm, which we denote by $\operatorname{det}_{S}(x)$, as having the properties that $\operatorname{det}_{S}(x) \in K$ and $\operatorname{det}_{S}(x y)=\operatorname{det}_{S}(x) \operatorname{det}_{S}(y)$. The notation is explained by the fact that if $F$ is a splitting field of $S$, that is, $S \otimes_{k} F \cong M_{n}(F)$, the $n \times n$ matrix ring over $F$, then $\operatorname{det}_{S}(x)=\operatorname{det}(x \otimes 1)$, where $\operatorname{det}()$ stands for the standard determinant function on $M_{n}(F)$. For a standard reference see [4, pp. 274 - 275].

Let $B$ be a prime PI ring with center $Z$. Then $Q(B)=B Q(Z)$ is a central simple finite dimensional $Q(Z)$-algebra, and by abuse of notation we write $\operatorname{det}_{B}(x)$ for $\operatorname{det}_{Q(B)}(x)$.

Theorem 2.3. Let $S, R$ be $P I$ rings, and let ${ }_{S} M_{R}$ be a bimodule. Let $Z=Z(R)$. Assume that:

(1) $M_{R}$ is a finitely generated and torsion-free R-module,

(2) ${ }_{S} M$ is a finitely generated and faithful $S$-module,

(3) $R$ is a prime Noetherian PI ring, and $S$ is a left Noetherian PI ring.

Then $A \equiv \operatorname{End}_{Z}\left(M_{Z}\right)$ is prime Noetherian and a finite $R_{r}$-module, as well as a finite $S_{l}$-module. Moreover $S$ is right Noetherian, $Q(S)$ exists and ${ }_{S} M$ is torsionfree.

Note: Here $R_{r}=\left\{x_{r} \mid x \in R\right\}$, where $x_{r}$ is the right multiplication by $x$ on $M$, and $S_{l}=\left\{y_{l} \mid y \in S\right\}$, where $y_{l}$ is the left multiplication by $y$ on $M$.

Proof. By Corollary 2.2, $A$ is a prime PI ring which contains both $R_{r}$ and $S_{l}$. We shall consider the action of $A$ on $M$ on the right, implying that $M$ is a right $A$-module. Also $M_{R}$ is finitely generated and hence $M_{R_{r}}$ is finitely generated, which shows that $M_{A}$ is finitely generated. Also $A \equiv \operatorname{End}_{Z}(M)$ implies that $M_{A}$ is faithful. Therefore by the $H$-condition (e.g. [7, Prop. 8.7]) $A_{A} \hookrightarrow M_{A}^{n}$, for 
some $n$. Since $R_{r} \subseteq A$ we also have $A_{R_{r}} \hookrightarrow M_{R_{r}}^{n}$, which implies that $A$ is a finite $R_{r}$-module, and in particular $A$ is Noetherian (from both sides) and Schelter integral over $R_{r}$. Similarly $A_{S_{l}} \hookrightarrow M_{S_{l}}^{(n)}$ shows, since $S_{l}$ is right Noetherian (since $\left.S_{l} \cong S^{0}\right)$, that $A_{S_{l}}$ is a right finitely generated $S_{l}$-module, and since $S_{l}$ is right Noetherian, we get that $A$ is also Schelter integral over $S_{l}$. Now by [5], $S_{l}$ is also left Noetherian and consequently $S$ is also right Noetherian. Now since $A$ is Schelter integral over $S_{l}$, then by [2, Theorem 7.1] $\mathcal{C}_{S_{l}}\left(N i l\left(S_{l}\right)\right) \subseteq \mathcal{C}_{A}(0)$ and in particular $\mathcal{C}_{S_{l}}\left(N i l\left(S_{l}\right)\right) \subseteq \mathcal{C}_{S_{l}}(0)$. This shows that $S_{l}$ has an Artinian quotient ring and the same holds for $S$.

We shall next show that $M_{A}$ is torsion-free. It suffices to show that each $\delta \neq$ $0 \in Z(A)$ acts regularly on $M$ (since if $a$ in $A$ is regular, then $\operatorname{det}_{A}(a) \in Q(Z(A))$ is non-zero, and so $\exists c \in Z(A) \neq 0$ such that $\left.\operatorname{cdet}_{A}(a) \in Z(A)\right)$.

We shall first observe that $Z\left(R_{r}\right) \subseteq Z(A)$. Indeed if $\theta \in A, z \in Z(R)$, then $m .\left(\theta \cdot z_{r}\right)=\theta(m) z=\theta(m z)=m .\left(z_{r} \cdot \theta\right)$, for each $m \in M$; that is, $\theta z_{r}=z_{r} \theta$, for each $\theta \in \operatorname{End}_{Z}(M) \equiv A$. Therefore if $K \equiv Q\left(Z\left(R_{r}\right)\right)$, then $Q\left(R_{r}\right)=K R_{r} \subseteq$ $K A \subseteq Q(Z(A)) A=Q(A)$. Now $A$ is integral over $R_{r}$, and therefore $K A$ is integral over $K$ and in particular $\delta$ is integral over $K$. So there exist $c_{0}, \ldots, c_{n} \in Z\left(R_{r}\right)$, $c_{n} \neq 0$, satisfying $c_{0} \delta^{n}+c_{1} \delta^{n-1}+\ldots+c_{n}=0$. Then $m \delta=0$ implies $m c_{n}=0$, and $m=0$ by the torsion-freeness of $M_{R}$. Finally, if $t m=0$, for some $t \in \mathcal{C}_{S}(0)$, then $m t_{l}=0$, but $t_{l} \in \mathcal{C}_{S_{l}}(0) \subseteq \mathcal{C}_{A}(0)$, and $m=0$ by the torsion-freeness of $M_{A}$.

The next result is a surprising corollary.

Theorem 2.4. Let ${ }_{S} M_{R}$ be a bimodule which is finitely generated and torsion-free from both sides, where $S$ is a left Noetherian PI and $R$ is a prime Noetherian PI ring. Then there exists a centralizing extension $B$ of both $R_{r}$ and $S_{l}$. Moreover $B$ is finitely generated over both.

Proof. Let $Z=Z(R)$ and $A=\operatorname{End}_{Z}\left(M_{Z}\right)$ be as in Theorem 2.3. Then $B \equiv S_{l} \cdot R_{r}$ is a centralizing extension of both $S_{l}$ and $R_{r}$. Moreover since by the previous theorem $A$ is a finite $S_{l}$-module as well as a finite $R_{r}$-module and both are Noetherian, we conclude that $B$ is finite over both.

Remark 2.5. The previous result suggests that $S p e c R \cong S p e c R_{r}$ is closely connected to $\operatorname{Spec} S \cong \operatorname{Spec} S^{0} \cong \operatorname{Spec}_{l}$. This seems to be new even in the finite extension case $R \subset S$.

Corollary 2.6. Let $R$ be a prime Noetherian PI ring with center $Z(R) \equiv Z$. Then $\operatorname{End}_{Z}(R)$ is a prime Noetherian PI ring, which is a finite $R_{r}$-module as well as a finite $R_{l}$-module.

Remark 2.7. This can be used to give a different proof of [1, Theorem 1.8].

The next result is our main tool in generalizing [1, Theorem 1.8].

Proposition 2.8. Let $R$ be a prime Noetherian PI ring with center $Z$, and let $M$ be an R-bimodule which is finitely generated torsion-free (from both sides). Let $\varphi, \psi \in \operatorname{End}_{Z} M \equiv A$ satisfy:

(1) $\operatorname{det}_{A} \varphi=\operatorname{det}_{A} \psi \neq 0$,

(2) $\varphi(M) \subseteq \psi(M)$.

Then $\varphi(M)=\psi(M)$. 
Proof. We can clearly extend $\psi$ to $\operatorname{End}_{K}(M K)$, where $K=Q(Z)$ is the quotient field of $Z$. Then $\operatorname{det} \psi \neq 0$ implies that $\psi$ has an inverse $\psi^{-1} \in E n d_{K}(M K)$. Hence (2) implies that $\alpha \equiv \psi^{-1} \circ \varphi \in \operatorname{End}_{Z} M \equiv A$. Moreover,

$$
\operatorname{det}_{A} \alpha=\left(\operatorname{det}_{A} \psi^{-1}\right) \operatorname{det}_{A} \varphi=\left(\operatorname{det}_{A} \psi\right)^{-1} \operatorname{det}_{A} \varphi=\left(\operatorname{det}_{A} \varphi\right)^{-1}\left(\operatorname{det}_{A} \varphi\right)=1 .
$$

Hence, by the Cayley-Hamilton equation, $\alpha$ has an inverse $\alpha^{-1} \in T(A)$, the trace ring of $A$. Now by Theorem $2.3 A$ is a prime Noetherian PI ring and consequently $T(A)$ is a finite centralizing extension of $A$. Let $\left(\alpha^{-1}\right)^{m}+a_{1}\left(\alpha^{-1}\right)^{m-1}+\ldots+a_{m}=0$ be an integral equation of $\alpha^{-1}$ over $A$, with $a_{1}, \ldots, a_{m}$ in $A$. Multiplying this equation by $\alpha^{m-1}$ yields that $\alpha^{-1} \in A$. In particular $\alpha$ is an onto map. Since $\varphi=\psi \circ \alpha$, this shows that $\varphi(M)=\psi(M)$.

Lemma 2.9. Let $S$ be a central simple finite dimensional $K$-algebra. Then $T \equiv$ $\operatorname{End}_{K}(S)$ is a central simple $K$-algebra. Moreover, for each $x \in S$ we have $\operatorname{det}_{T}\left(x_{l}\right)$ $=\operatorname{det}_{S_{l}}\left(x_{l}\right)^{n}=\operatorname{det}_{S}(x)^{n}=\operatorname{det}_{S_{r}}\left(x_{r}\right)^{n}=\operatorname{det}_{T}\left(x_{r}\right)$, where $n^{2}=[S: K]$.

Proof. This appears for example in [4, pp. 274-275].

Lemma 2.10. Let $R$ be a prime Noetherian PI ring, and let $M$ be a $Z$-central $R$ bimodule which is finitely generated and torsion-free from both sides. Let $K \equiv Q(Z)$ be the quotient field of $Z$, and let $Q(R)$ be the quotient ring of $R$. Then ${ }_{Q(R)} K M$, $K M_{Q(R)}$ are free and $\operatorname{rank}_{R} M=\operatorname{rank} M_{R}$.

Proof. Clearly $K M=M K$ and $Q(R)=K R=R K$. Now $K M$ is a completely reducible left module over $Q(R) \otimes_{K} Q(R)^{\circ} \cong \operatorname{End}_{K}\left(Q(R)^{\circ}\right.$, since the latter is a simple central $K$-algebra. Consequently $K M \cong \underbrace{V \oplus \cdots \oplus V}_{\text {t-times }}$, where $V$ is the unique irreducible left $Q(R) \otimes_{K} Q(R)^{\circ}$ module. Clearly $Q(R)$ is an irreducible $Q(R) \otimes_{K} Q(R)^{\circ}$-module. Therefore $V \cong Q(R)$ implies that ${ }_{Q(R)} V \cong_{Q(R)} Q(R)$, showing that $K M$ is a free left $Q(R)$-module with $\operatorname{rank}_{Q(R)} K M=t$. Similarly using $V_{Q(R)} \cong Q(R)_{Q(R)}$, we show that $\operatorname{rank} K M_{Q(R)}=t$ and $K M_{Q(R)}$ is free.

Lemma 2.11. Let $R$ be a prime Noetherian PI ring with center $Z$. Let $M$ be a finitely generated torsion-free $Z$-central $R$-bimodule. Then for every $x \in \mathcal{C}_{R}(0)$, we have $\operatorname{det}_{A}\left(x_{r}\right)=\operatorname{det}_{R}^{t n}(x)=\operatorname{det}_{A}\left(x_{l}\right)$, where $A=\operatorname{End}_{Z} M, t=\operatorname{rank}_{R} M$ and $n=P I . \operatorname{deg} R$.

Proof. By Lemma 2.10, $M K=K M$ is a free left $Q(R)$-module of rank $t$. Hence $K M=Q(R) u_{1} \oplus \ldots \oplus Q(R) u_{t}$, as left $Q(R)$-modules. Hence $\operatorname{End}_{K}(M K) \cong$ $M_{t}\left(\operatorname{End}_{K} Q(R)\right)$. Moreover, since $\left.x_{l}\left(Q(R) u_{i}\right)\right) \subseteq Q(R) u_{i}$, for each $i=1, \ldots, t$, then the matrix representing $x_{l} \in \operatorname{End}_{K}(M K)$ is given by the $t \times t$ matrix:

$$
x_{l}=\left(\begin{array}{cccc}
x_{l, Q} & 0 & \cdots & 0 \\
0 & x_{l, Q} & \cdots & 0 \\
\vdots & \vdots & \ddots & \vdots \\
0 & 0 & \cdots & x_{l, Q}
\end{array}\right),
$$

where $x_{l, Q}$ is the matrix representing left multiplication by $x$ on $Q(R)$.

Consequently $\operatorname{det}_{A}\left(x_{l}\right)=\operatorname{det}_{T}^{t}\left(x_{l, Q}\right)$, where $T=\operatorname{End}_{K}(Q(R))$. Let $S=Q(R)$; then by Lemma 2.9 we have $\operatorname{det}_{T}\left(x_{l, Q}\right)=\operatorname{det}_{S}^{n}(x)$. Consequently $\operatorname{det}_{A}\left(x_{l}\right)=$ $\operatorname{det}_{S}^{t n}(x)$. The other equality follows by a similar argument on the right side using Lemma 2.10. 
Recall that it is proved in [1, Theorem 1.8] that $x R \subseteq R x$ implies $x R=R x$, for each prime Noetherian PI ring $R$. The following extension of this result to $R$-bimodules is obtained by an entirely different proof.

Theorem 2.12. Let $R$ be a prime Noetherian PI ring with center $Z$. Let $M$ be a finitely generated torsion-free (on both sides) $R$-bimodule which is $Z$-central. Suppose that $x M \subseteq M x$, for some $x \in R$. Then $x M=M x$.

Proof. We clearly may assume that $x \neq 0$. We shall next show that $x \in \mathcal{C}_{R}(0)$. Indeed the torsion-freeness of ${ }_{R} M$ implies that ${ }_{R} M$ is faithful and hence $x M \neq 0$. Suppose $x y=0$ for some non-zero $y \in R$. Then $y \in r-a n_{R} x M$, a non-zero two-sided ideal in $R$, implying the existence of a non-zero $c \in Z \cap\left(r-a_{R} n_{R} x\right)$. Hence $c x M=x M c=0$ shows that $c x=0$ and therefore $x=0$, which was excluded. Let $\varphi=x_{l}, \psi=x_{r}$. Then $x M \subseteq M x$ is equivalent to $\varphi(M) \subseteq \psi(M)$. Also by Lemma $2.11 \operatorname{det}_{A}\left(x_{r}\right)=\operatorname{det}_{A}\left(x_{l}\right) \neq 0$, where $A \equiv \operatorname{End}_{Z} M$. So all the conditions of Proposition 2.8 are in place and its conclusion $\varphi(M)=\psi(M)$ is the required result.

Apparently, even the next corollary seems to be new.

Theorem 2.13. Let $R$ be a prime Noetherian PI ring with $x I \subseteq I x$, for some two-sided ideal $I \subseteq R$ and $x \in R$. Then $x I=I x$.

Question 2.14. Is there a non-normal element $x \in R$ satisfying $x I=I x$, for some non-zero ideal $I \subseteq R$, where $R$ is a prime Noetherian PI ring?

The next result provides a negative answer to Question 2.14 in the case where $R$ is in addition a maximal order.

Lemma 2.15. Let $R$ be a prime Noetherian PI maximal order and $x I=I x$ for some non-zero ideal $I$ in $R$ and $x \in R$. Then $x$ is a normal element in $R$.

Proof. If $x y=0$, then $x I y=0$, showing, since $x \neq 0$, that $y=0$. Therefore $x$ is a regular element in $R$ and consequently $x^{-1} \in Q(R)$. We have

$$
\left(x R x^{-1}\right) I=x R\left(x^{-1} I x\right) x^{-1}=x R I x^{-1}=x I x^{-1}=I .
$$

Therefore $x R x^{-1} \subseteq O_{l}(R) \equiv\{t \in Q(R) \mid t I \subseteq I\}$ (see e.g. 99, 3.1.12]). Thus since $R$ is a maximal order, $O_{l}(R)=R$ (e.g. [9, 5.1.4]). Therefore $x R x^{-1} \subseteq R$, or equivalently $x R \subseteq R x$. Hence by [1, Proposition 1.8] or Theorem A, $x R=R x$ and $x$ is a normal element in $R$.

A similar argument to the one appearing in Theorem 2.12, using Proposition 2.8, proves the following result.

Theorem 2.16. Let $R, M$ be as in Theorem 2.12 and $x, y$ be regular elements in $R$. Suppose that $x M y \subseteq y M x$. Then $x M y=y M x$.

Question 2.17. It is easily verified that $r-\operatorname{ann}(x) \subseteq r-\operatorname{ann}(y)$ and $l-a n n(y) \subseteq$ $l-\operatorname{ann}(x)$. Thus we merely have to assume in Theorem 2.16 that one of $\{x, y\}$ is regular. Can one avoid it altogether? Even the special case $x R y \subseteq y R x$ appears to be open. 


\section{REFERENCES}

[1] A. Braun and C. R. Hajarnavis, Generator ideals in Noetherian P.I. rings, J. Algebra 247 (2002), 134-152. MR.1873387 (2003a:16033)

[2] A. Braun and V. Vonessen, Integrality for P.I. rings, J. Algebra 151 (1992), 39-79. MR.1182014 (94g:16034)

[3] A. Braun and R. B. Warfield, Jr., Symmetry and localization in Noetherian prime P.I. rings, J. Algebra 118 (1988), 322-335. MR969675 (89k:16031)

[4] P. M. Cohn, Algebra, III, Wiley, New York and Chichester, 1991. MR.1098018 (92c:00001)

[5] B. Cortzen and L. W. Small, Finite extensions of rings, Proc. Amer. Math. Soc. 103 (1988), 1058-1062. MR:954983 (89f:16020)

[6] A. Frölich, I. Reiner and S. Ullom, Class groups and Picard groups of orders, Proc. London Math. Soc. 29 (1974), 405-434. MR0357464 (50:9932)

[7] R. R. Goodearl and R. B. Warfield, An Introduction to Noncommutative Noetherian Rings, London Math. Soc. Student Texts, 16, Cambridge University Press, 1989. MR1020298 (91c:16001)

[8] R. M. Guralnick, J. C. Robson and L. W. Small, Normalizing elements in PI rings, Proc. Amer. Math. Soc. 123 (1995), 1955-1957. MR.1301026 (95h:16032)

[9] J. C. McConnell and J. C. Robson, Noncommutative Noetherian Rings, Wiley, New York, 1987. MR934572 (89j:16023)

[10] S. Montgomery, A generalized Picard group for prime rings, in Banach Center Pub. No. 26, pp. 55-63, Polish Acad. Sci., Warsaw, 1990. MR.1171225 (93g:16026)

[11] L. H. Rowen, Ring Theory, Academic Press, San Diego, 1988. MR1095047 (94e:16001)

[12] W. V. Vasconcelos, On quasi-local regular algebras, in "Sympos. Math XI", pp. 11-12, Academic Press, London, 1973. MR0330159 (48:8497)

Department of Mathematics, University of Haifa, Haifa, Israel 31905

E-mail address: abraun@math.haifa.ac.il 